\title{
Field of Energy
}

\author{
Armando T. Canero*, Marco A. Canero** \\ *Departamento de Física, Facultad de Ingeniería, Universidad de Buenos Aires \\ **Facultad de Ciencias Exacta y Naturales, Universidad de Buenos Aires \\ armandotcanero@yahoo.com.ar
}

\section{ABSTRACT}

The study of physics requires the definition of general characteristics such as the so-called fundamental properties of space and time, which are homogeneity and isotropy. From the application of the homogeneity of time in the integral equations of the movement arises the theorem of the conservation of energy. That the parameter of variation be time leads to defining energy as scalar.

Relativistic mechanics has shown that time is one of the dimensions of a tetra-dimensional space and, therefore, an event is projected in the spatial and temporal dimensions, this projection varies according to the reference system that is used. This indicates that equating time to a dimension of space, should be analyzed not only under the condition of homogeneity but also of the isotropy. This leads to analyzing energy as a vector.

In classical mechanics, a body moving in a gravitational field its energy can be decomposed in two directions, one that remains constant, normal to the field, and the other that varies with gravity. This shows vector properties of energy.

This study proposes a more general response through the energy field.

\section{INDEXING TERMS/KEYWORDS}

Energy, work, homogeneity, isotropy.

\section{ACADEMIC DISCIPLINE AND SUB-DISCIPLINES}

Physics, theoretical Physics.

\section{SUBJECT CLASSIFICATION}

Physics classification.

\section{TYPE (METHOD/APPROACH)}

Theoretical Analysis.

Language: English

Date of Publication: 30-07-2018

DOI: 10.24297/jap.v14i2.7491

ISSN: 2347-3487

Volume: 14 Issue: 2

Journal: JOURNAL OF ADVANCES IN PHYSICS

Website: https://cirworld.com

This work is licensed under a Creative Commons Attribution 4.0 International License. 


\section{INTRODUCTION}

The energy taken as a scalar allows the resolution of a large number of problems, but hinders the physical compression of many others. The example of the projectile movement highlights not only that the anisotropy of the medium is an element that define the vector condition of the physical property, that from here in advance it will be mentioned as an energy field, and of which it will be said that the energy is its module. The importance of the study of the scalar magnitude called energy is not discuss, but it is emphasized that the complete physical properties in question have the character of a vector and that if it is not studied in its entirety, it generates confusion and misinterpretations.

In the projectile movement, or of the pendulum, or the body with electric charges in a magnetic field, it is found that the energy is related to a field where the directions are not commutable (neither the sense), which determine the vector property of the energy field. Examples are:

- The gravitational potential energy is related to the distribution of the masses.

- The electric potential energy is determined by the distribution of charges.

- The kinetic energy is defined through the speed of the reference system which is called inertial and links to the rest of the universe (laboratory, Earth, fixed stars).

This supports the proposal of an energy field as a physical medium from which energy can be taken as its module.

If it is analyzed from the point of view of mathematics, the equations used for the definition of energy find that there is an internal product in them, which results in a norm that determines a distance and therefore defines a metric; this implies that this is a vector space.

\section{METHODOLOGY}

The analysis will be applied first from the physical aspect and then from the mathematician, to demonstrate of vector features energy field. It will begin with the kinetic energy, following with other kinds of energies and it will be finished with the work.

\section{Kinetic energy, analysis:}

From the point of view of physics. In the projectile movement when calculating the maximum height from energetic conditions, it is done by equating the kinetic energy in the vertical axis with the potential energy, and keeping the horizontal kinetic energy constant. Here we can clearly see the dependence of the direction and the sense, of the kinetic energy field. The algebraic sum of the two components, in the axes $x$ and $y$, gives the value of the total energy field whose module is the kinetic energy, keep in mind that this responds to a vector sum over a space of norm L1. So it must be concluded that the kinetic energy field is vector.

From the mathematical point of view, considering that every internal product induces a single norm and that the equation that determines the kinetic energy is given by the expression $\mathbf{E}_{\mathrm{c}}=\frac{1}{2} \mathrm{~m} \mathbf{v}$. $\mathbf{v}$, this internal product determines a metric, whose norm, for this case, it is L1. See Bonilla-Barraza [1], Thompson, K. [2]. So we have a linear space $\mathbf{E}$ (on the body of real numbers), which has a rule defined that every pair of vectors $\mathbf{E}$ assigned a real number, called internal product, which satisfies the following axioms:

$\forall \mathrm{x}, \mathrm{y}, \mathrm{z}, \in \mathbf{E} \mathrm{y} \forall \alpha, \beta \in \mathrm{R}$

Linear with respect to the second argument $(\mathrm{z}, \alpha \mathrm{x}+\beta \mathrm{y})=\alpha(\mathrm{z}, \mathrm{x})+\beta(\mathrm{z}, \mathrm{y})$

Hermitian (symmetric in a space from real $(\mathrm{Y}, \mathrm{X})=(\mathrm{X}, \mathrm{Y}))$

Defined positive $(x, x) \geq 0 y(x, x)=0 \leftrightarrow x=0$, where $\mathbf{0} \in \mathbf{E}$ is the null vector of that space. 
This implies that it is a normed space and therefore it is a K-vector space E, provided with a norm, see Burago [3]. With which it is concluded that the kinetic energy field is a vector.

\section{Elastic potential energy, analysis:}

From the point of view of physics. In the calculations with the elastic energy this magnitude is taken as a scalar, however this energy can only be restored or transferred in a single direction, that of compression of the spring. It is cannot get work ninety degrees from this address. The physical property thus stored has a magnitude, an address and a sense, therefore, the energy is the module of a vector, so it must be introduce the concept of field of elastic potential energy.

From the mathematical point of view, just as for the kinetic energy it is seen that the equation $\mathbf{E}_{\mathrm{c}}=\frac{1}{2} \mathrm{k} \mathbf{x}$. $\mathbf{x}$, it has an internal product therefore this induces a norm. This norm is again the $L 1$, and due to the same considerations of the kinetic energy field, the need to define the elastic potential energy as the module of a vector.

\section{Gravitational potential energy, analysis:}

From the point of view of physics, as in the two previous cases, the magnitude has a direction and sense of application; it cannot be transformed or used interchangeably. The fact that it is a potential function does not imply that the magnitude in question is not vector. Again, it is the case that the gravitational potential energy is the module of a vector gravitational potential energy field. Keep in mind that this is the field that was taken as an example to denote that the kinetic energy field is a vector.

From the mathematical point of view, as for the previous cases, we see that the equation $\mathbf{E}_{\mathrm{p}}=\mathrm{m} \mathbf{g}$. $\mathbf{h}$, has an internal product, therefore this induces a norm. For this particular expression the product has two apparently different magnitudes $\mathrm{g}$ and $\mathrm{h}$, this does not change the result as can be seen

$$
\text { g. } h=\frac{d^{2} x}{d t^{2}} \cdot \mathbf{x}
$$

If it is taken into account that the derivation operator is linear and therefore the order with respect to the product of the distances can be inverted and considering that

$$
\frac{\mathrm{d}^{2} \mathbf{x}^{2}}{\mathrm{dt}^{2}}=\frac{\mathrm{d}}{\mathrm{dt}}\left(\frac{\mathrm{dx}^{2}}{\mathrm{dt}^{2}}\right)=\frac{\mathrm{d}}{\mathrm{dt}}\left(2 \mathrm{x} \cdot \frac{\mathrm{dx}}{\mathrm{dt}}\right)=2\left(\frac{\mathrm{dx}}{\mathrm{dt}}\right)^{2}+2 \mathbf{x} \frac{\mathrm{d}^{2} \mathbf{x}}{\mathrm{dt}^{2}}
$$

Remains

$$
\mathbf{g} \cdot \mathbf{h}=\frac{1}{2} \frac{\mathrm{d}^{2}}{\mathrm{dt}^{2}}(\mathbf{x} \cdot \mathbf{x})-(\mathbf{v} \cdot \mathbf{v})
$$

As it can be seen, this is an expression that involves the internal product that determines a vector space of norm L1.

Work, analysis:

From the physical point of view. It represents the transfer of energy, its units are the same, therefore it must be expected that its behavior will be the same.

The work is defined as a scalar in accordance with the energy, but the concept that is really applied is that of modulo of the transferred energy field. It is emphasized that although the module is a number like a scalar, it is a component part of a vector and for the physical aspects it is not independent of this. It is to be noted that the energy field maintains its direction of transformation, that is, this work converted into energy cannot be transformed later in any direction. The physical concept of work does not require a vector field since that role it is fulfilled by the energy field. 
From the mathematical point of view, following the same steps as for the gravitational potential energy.

$$
\int \mathbf{F} \cdot \mathrm{d} \mathbf{x} \cong \mathbf{F} \cdot \mathbf{x}=\mathrm{m} \frac{\mathrm{d}^{2} \mathbf{x}}{\mathrm{dt}^{2}} \cdot \mathbf{x}=\frac{1}{2} \mathrm{~m} \frac{\mathrm{d}^{2} \mathbf{x}^{2}}{\mathrm{dt}^{2}}=\frac{1}{2} \frac{\mathrm{d}^{2}}{\mathrm{dt}^{2}}(\mathbf{x} \cdot \mathbf{x})
$$

It shows that the formula that represents the work admits a vector representation and that it is defined in this work as the energy field.

\section{Elements concurrent to the concept of energy field as vector magnitude}

The Pointing vector is the energy transferred by the electromagnetic wave. Its vector character follows directly from its formula, since it emerges as the external product of two vectors. Here the energy has not been defined as the module but as the vector. To compare why one is defined as a scalar and the other as a vector arises that the reason is based on the kind of multiplication used, whether the internal or external product and not of analyze the real properties of the physical event. From this it is concluded that the magnitude in analysis is vector and when it is calculated through the internal product it is the magnitude module, to which it is be must add the direction and the sense to define it completely.

In the propagation of sound as transverse waves, see Canero [4], the energy transfer is presented again as an external product of two vectors, which gives the sound energy a vector character, in the same way that the vector of Pointing

\section{ANALYSIS}

In this point the concepts expressed in the previous paragraphs will be applied to specific problems.

\section{Shock}

One of the most used applications of energy expressions is given in the problems of shock, so it is necessary to validate the new perspective with the experience harvested in this topic.

In the collisions between two bodies it is usual to differentiate between elastic shock and plastic. The difference between the two can be summarized as the first conserves energy and the second does not. This does not contribute to the understanding of the problem and restricts the understanding of it, being a difference by definition and not by interpretation of the events. For this reason, the following differentiation between the two is proposed.

Elastic shock is what keeps:

- Total energy and energy field relative to the center of mass*.

- Total amount of movement ** and relative to the center of mass.

Plastic shock is what keeps only:

- Energy field relative to the center of mass.

- Amount of movement (relative to the center of mass).

* With the energy field relative to the center of mass the vector sum of the energy field must be realized.

** The total amount of movement must be interpreted to the sum of the modules of the individual movement amount of each body.

That is to say two aspects must be considered one that can be called external energy, which is the one that has the center of mass; and an internal energy, which it is what can be annulled by acting the body of the system on each other. This can be seen in the completely plastic clash where the two bodies end up stuck to 
one another, which indicates that there is no energy separating them. This division between internal and external can also be applied for the amount of movement, with the total amount of movement and the relative to the center of mass.

Shock calculation:

$\boldsymbol{r}_{a}+\boldsymbol{r}_{b}=\boldsymbol{r}_{1}-\boldsymbol{r}_{2}$

Being $\boldsymbol{r}_{a}$ and $\boldsymbol{r}_{b}$ the position vectors of the masses referred to the center of mass. See fig 1 .

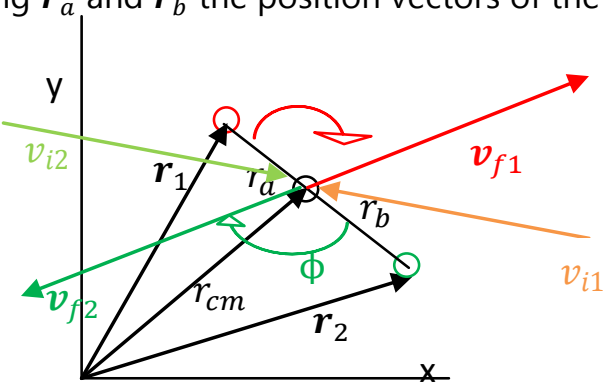

Fig 1: Diagram of speeds referred to the center of mass.

$\boldsymbol{r}_{c m} m_{c m}=\sum \boldsymbol{r}_{1} m_{1}$

$\boldsymbol{r}_{c m}=0 \rightarrow \sum \boldsymbol{r}_{i} m_{i}=0$

$\boldsymbol{r}_{1} m_{1}=\boldsymbol{r}_{2} m_{2}$

The collision seen from the center of mass imposes that the angle of deviation of the bodies be the same, since the center of mass must always be between the two bodies. That is, $\varnothing=\delta$.

Shock between two bodies, the bi-dimensional problem is analyzed, following the traditional method. It is proposed: conservation of quantity of movement in a system solidary to the center of mass in an instant of time and conservation of energy between the before and after the impact. From here the system of equations that remains is the following.

$\boldsymbol{P}_{1 x}+\boldsymbol{P}_{2 x}=m_{1} \boldsymbol{v}_{f x 1}+m_{2} \boldsymbol{v}_{f x 2}=0$

$\boldsymbol{P}_{1 y}+\boldsymbol{P}_{2 y}=m_{1} \boldsymbol{v}_{f y 1}+m_{2} \boldsymbol{v}_{f y 2}=0$

$E_{i}-E_{f}=\frac{m_{1}}{2} v_{i 1}{ }^{2}+\frac{m_{2}}{2} v_{i 2}{ }^{2}-\frac{m_{1}}{2} v_{f 1}{ }^{2}-\frac{m_{2}}{2} v_{f 2}{ }^{2}$

The velocities in the directions $x$ and $y$ are expressed as a function of the total velocity and its projection in each direction. This and the fact that the system is solidary to the center of mass allows to pass from a system of four unknowns, the velocities: $\boldsymbol{v}_{f 1 x}, \boldsymbol{v}_{f 1 y}, \boldsymbol{v}_{f 2 x}, \boldsymbol{v}_{f 2 y}$, to one of three unknowns: two velocities and an angle $\boldsymbol{v}_{f 1}, \boldsymbol{v}_{f 2}, \alpha$. Where $\alpha$ is the angle between the vector momentum and the vertical axis.

$m_{1} \boldsymbol{v}_{f 1} \cos \alpha+m_{2} \boldsymbol{v}_{f 2} \cos \alpha=0$

$m_{1} \boldsymbol{v}_{f 1} \operatorname{sen} \alpha+m_{2} \boldsymbol{v}_{f 2} \operatorname{sen} \alpha=0$

$m_{1} v_{i 1}^{2}+m_{2} v_{i 2}^{2}-m_{1} v_{f 1}^{2}-m_{2} v_{f 2}^{2}=0$

It is seen that equations (12) and (13) are equal so one of them must be discarded, it can also be seen that there are only two unknowns left, since the angle in the equations has disappeared. 
It should be noted that equations (9) and (10) represent the conservation of the amount of movement with respect to the center of mass. That is, the conserved magnitude is not the amount of individual movement of each body, but the amount of movement of the center of mass. And for this condition, the energy of the center of mass is conserved as well as the amount of movement, both in integral form and in any of its projections. This is so given that for the center of mass there is no shock and therefore neither its amount of movement nor its energy can be altered. The condition of the center of mass is that of the relation of the set with the external environment. This indicates that equation (11) or (14) can be replaced by an equation that takes into account the conservation of the total momentum of the system, ie the sum of the absolute value of the momentum of all the bodies of the system. So the equation can be written as:

$\mathrm{m}_{1}\left|\mathbf{v}_{\mathrm{i} 1}\right|+\mathrm{m}_{2}\left|\mathbf{v}_{\mathrm{i} 2}\right|-\mathrm{m}_{1}\left|\mathbf{v}_{\mathrm{f} 1}\right|-\mathrm{m}_{2}\left|\mathbf{v}_{\mathrm{f} 2}\right|=0$

of the equation (12)

$\mathbf{v}_{\mathrm{f} 1}=\frac{\mathrm{m}_{2}}{\mathrm{~m}_{1}} \mathbf{v}_{\mathrm{f} 2}$

Substitute this expression 16 into 15

$v_{f 1}=\frac{m_{1} v_{i 1}+m_{2} v_{i 2}}{2 m_{2}}$

If you take into account that the coordinate system moves together with the center of mass, it is can write

$\mathrm{m}_{1} \mathbf{v}_{\mathrm{i} 1}+\mathrm{m}_{2} \mathbf{v}_{\mathrm{i} 2}=0$

Solving the equation for $\mathbf{v}_{\mathbf{i} 1}$ and plugging this into the (17)

$\mathbf{v}_{\mathrm{f} 2}=\mathbf{v}_{\mathrm{i} 2}$

Which must be fulfilled since the shock is elastic and is being analyzed from the center of mass. This same result can be found if (12) and (14) are used instead of equations (12) and (15).

$m_{1} \mathbf{v}_{\mathrm{i} 1}{ }^{2}+\mathrm{m}_{2} \mathbf{v}_{\mathrm{i} 2}{ }^{2}-\mathrm{m}_{1} \mathbf{v}_{\mathrm{f} 1}{ }^{2}-\mathrm{m}_{2} \mathbf{v}_{\mathrm{f} 2}{ }^{2}=0$

Keep in mind that the fact of being squared has the same effect as taking the absolute value of the number. If the ec (16) is introduced in (15) and it is regrouped, it remains:

$\mathrm{v}_{\mathrm{f} 2}=\sqrt{\frac{\mathrm{m}_{1} \mathbf{v}_{\mathbf{i}}{ }^{2}+\mathrm{m}_{2} \mathrm{v}_{\mathrm{i} 2}{ }^{2}}{\frac{\mathrm{m}_{2}{ }^{2}}{\mathrm{~m}_{1}}+\mathrm{m}_{2}}}$

Introducing the ec (18) one has again

$v_{\mathrm{f} 2}=\mathbf{v}_{\mathrm{i} 2} \sqrt{\frac{\frac{\mathrm{m}_{2}{ }^{2}}{\mathrm{~m}_{1}+\mathrm{m}_{2}}}{\frac{\mathrm{m}_{2}^{2}}{\mathrm{~m}_{1}}+\mathrm{m}_{2}}}=\mathbf{v}_{\mathrm{i} 2}$

This shows that it is not a balance of momentum and energy that determines the conditions of the bodies after the shock; rather, it is a balance between internal properties and external properties.

The interaction between two bodies in an elastic shock is a process that maintains the modulus of its components, both for the amount of movement and for the energy field. Therefore the relationship that links the before and after the shock, is the balance of the modules; for this reason, the equation of energy for the temporal relation has been taken. Since being the module of the energy field (proposed in this publication) takes the required condition without having to analyze the causes of its behavior. 
From the above we can distinguish the energy of the center of mass of the internal energy, see Landau [5]. What allows to give a clearer definition to differentiate the elastic collision of the plastic. The elastic collision is the one that conserves its internal energy and the completely plastic collision is the one that its internal energy is totally annulled. This indicates that since there is no internal energy that allows one to separate from the other, the two bodies must remain together.

The third equation (fourth in the traditional approach) is usually extracted from a geometric consideration. In which it is taken into account that the shock does not occur between the centers of mass, but on the surfaces of the bodies. This indicates that in the collision can be separated into two movements; one in the direction of the center of mass and the remaining at ninety degrees. The perpendicular movement to the impact direction is not altered and therefore its speed is maintained constant. This component of velocity is what generates the angular momentum, which is an invariant. Only the frontal impact remains in the analysis for the collision, for which the calculation shows that there is no difference between the considerations made for the amount of movement and the energy field, both are vectors.

Invariance of energy

The proposal of a one internal energy field and an external energy field allows us to answer a question that is normally avoided perform, which is: If the energy is an invariant How it is energy related between two systems of references in relative movement one with respect to the other. To respond to this, it is usually necessary to resort to calculating the speed in the new reference system through the kinematics and subsequently get the energy. But this is not use the property that the energy it is an invariant.

The invariant magnitude between the systems is the internal energy field. The external energy field is that of the center of mass and depends on the chosen reference system, its transformation between the different systems is simple.

Relativity

In relativistic mechanics it is work with tetra-vectors, this imposes as magnitude the vector time. In the literature on the subject reference is made to this mathematical entity whit a mixed condition, and giving the temporal component the status of three-dimensional scalar, example Lanadu [6]. This speaks of there being components according to the three spatial directions, characteristic own of the vectors. For relativistic mechanics this aspect is marked because time does not flow in the same way for a body with velocity relative to the coordinate system. The same consideration applies to energy and therefore correspond to it three spatial components. Energy is not mentioned as a vector, since it is not projected in a Euclidean way, which is the way physical vectors work. Even so, mathematically they are vectors of norm L1.

\section{CONSEQUENCES}

The definition of the energy field, it is can use:

To be achieving a clearer read of physical processes, allowing giving a sense to the sequence of events.

For offer an interpretation that allows differentiating between plastic and elastic collision.

To be calculate the energy in another reference system.

\section{CONCLUSIONS}

The definition of energy as the invariant, in processes in which conservative forces participate, clashes with the fact that this is vector invariance before a change of reference system; being the energy defined as a scalar. Working only with the module simplifies some reasoning but prevents the power directly transform between two systems. 
It is can see, both in the problem of projectile movement and shock, the convenience of defining the energy field, as a vector entity. In this way it is can eliminate the inconveniences shown, when it is have to:

Decompose the energy in different directions.

Interpret the difference between elastic shock and plastic shock.

Link the physical magnitude of energy between different reference systems.

\section{ACKNOWLEDGMENTS}

This research is financially supported by Departamento de Física de la Facultad de Ingeniería de la Universidad de Buenos Aires.

\section{REFERENCES}

[1] Bonilla-Barraza D. Al fin de cuentas, ¿Qué es una recta en la Geometría del Taxista? -Revista Tumbaga, V. 2, N. 10, pp. 53-68, diciembre, 2014. ISSN 1909-4841. Online 2216-118x.

[2] Thompson, K. and Dray, T. Taxicab angles and trigonometry IIME Journal, Vol. 11, No. 2, pp 87- 96, 2000.

[3] Burago D, A. Course in Metric Geometry, Department of Mathematics, Pennsylvania State University.

[4] Canero. A.T. 2017. Soun as a Transverse Wave, ISSN2347-3487, Volume 13 Number1, Journal of Advances in $\mathrm{P}$.

[5] Landau y Lifshitz, Física Teórica, V1, Teoría physics.

[6] Landau y Lifshitz, Física Teórica, V2, Teoría Clásica de los Campos. 